\title{
NOTES
}

\section{A low-stress restraint procedure for EEG acquisition in awake rats}

\author{
CRAIG E. TENKE, MICHAEL BUDEK, \\ ROGER CHUNG, and LLOYD GILDEN \\ Queen's College, City University of New York, \\ Flushing, New York
}

A restraint procedure was developed to reduce movement-related artifacts during the acquisition of EEG data from unanesthetized, unparalyzed rats. It combines the low-stress properties of a cloth restraint bag with the improved immobility of a rigid restraint device. The spontaneous behavior of the rat facilitates both the induction of and the subsequent release from restraint.

The electrographic correlates of movement are sources of artifact in EEG studies in which unanesthetized, unparalyzed rats are used. Although restraint procedures serve to reduce many sources of variability related to free movement and uncontrolled sensory stimulation, the resulting preparation is generally stressed. When acutely enforced, restraint is known to produce gastric ulcers (Brodie \& Hanson, 1960) and to activate the adrenal cortex (Knigge, 1961).

In this laboratory, a reduction of struggling during restraint has previously been achieved by means of a series of drugged adaptation trials (Gilden, 1978; Gilden \& Kozakiewicz, 1976). Although stomach ulceration has not been detected, a decrease of struggling over sessions is variable, and at times slow. The procedure described here was developed to reliably minimize acute stressrelated behavior (e.g., struggling, vocalization) and movement-related electromechanical transients under less antagonistic conditions. It has been successfully applied to 12 adult male Long-Evans rats and has proved to be effective for EEG recordings during concurrent brainstem stimulation (Tenke, 1983).

The core of the restraint procedure is a cloth, funnelshaped restraint bag adapted from Bellingham (1980). Modifications include a pair of snaps and a drawstring in front of the Velcro-lined seam (shown on the left in Figure 1, top panel). The force applied by the front drawstring is distributed by virtue of a second pass around the outside of the bag through stabilizing loops constructed of cloth.

Rats readily enter the bag, and such behavior is maintained by allowing three runs through the bag (Velcro,

The authors' mailing address is: Department of Psychophysiology, N.Y.S. Psychiatric Institute, 722 W. 168th Street, New York, New York 10032. snaps, and drawstrings loosened) before and after each session. After the subject enters the bag during restraint periods (Velcro and snaps secured), a rear drawstring is immediately secured. Gentle manual pressure assures the emergence of the rat's head, after which the front drawstring is tightened and tied with a slipknot. This prevents the subject from withdrawing its head into the bag.

Once the subject has been secured in the restraint bag, a secondary device is required to maintain acceptable immobility. This device consists of a flat, padded base with a U-shaped, padded well to confine the subject's head (Figure 1, bottom panel). The bag enclosing the rat is secured onto this base by a series of cloth straps.

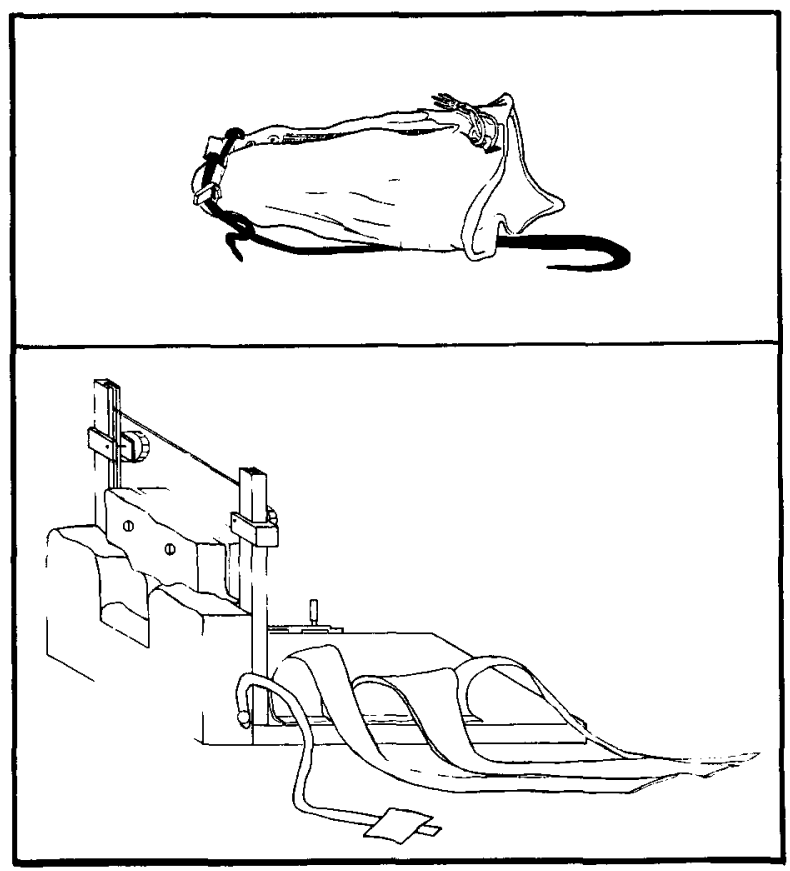

Figure 1. Top panel: Restraint bag with Velcro seam partially separated to show open snaps in front of it (left). The front drawstring may be seen emerging from a sleeve sewn into the fabric. Its second pass on the outside of the bag courses in front of the L-shaped metal stop and through a series of stabilizing loops (one visible at the top of the bag). The rear drawstrings and the scoop-like shape of the back of the bag are also apparent. Bottom panel: The rigid restraining device illustrated in orthogonal projection. The base of the device measures $26.5 \mathrm{~cm}$ in length. The transparent Plexiglas neck brace allows a view of the compression clamps and their associated adjustment knobs. A large block of foam rubber is bolted to the bottom of the brace. The retaining strap may be secured by use of the attached Velcro strip (shown). The three primary straps make use of a series of screw clamps (one shown). 
Once the subject has been strapped on to the base of the secondary device, a thickly padded, guillotine-like neck brace is lowered. Withdrawal of the head is prevented by the presence of a metal stop sewn onto the top of the bag, behind which a thin retaining strap is secured. Since experience has indicated that struggling is inversely related to the security and spatial distribution of the restraining influences, an Ace bandage is generally wrapped around the back of the fully restrained subject. After the recording electrode plugs have been connected, additional head padding is added.

To release the animal, the front drawstring is quickly untied, and the neck brace and stay are loosened. When the snaps are then unfastened, the typical rat spontaneously jumps forward and out of restraint. Even after $1.5 \mathrm{~h}$ of full restraint, subjects rarely balk at reentering the bag during the postrestraint runs.

In the current implementation, rats are briefly run through the bag without restraint daily throughout an initial adaptation period. Full restraint is enforced three times a week, and increased in duration from a few minutes to $1.5 \mathrm{~h}$. A total of seven sessions is typically re- quired, with some variation depending on the adaptability of the individual subject. This time course may undoubtedly be compressed considerably.

\section{REFERENCES}

Bellingham, W. P. An effective, cheap, and relatively nontraumatizing rat restrainer. Behavior Research Methods \& Instrumentation, 1980, 12, 79-80.

Brodie, D. A., \& Hanson, H. M. A. Study of the factors involved in the production of gastric ulcers by the restraint technique. Gastroenterology, 1960, 38, 353-360.

GiLden, L. Dual action effects of morphine on the electrical activity of the dorsal tegmentum. Pharmacology, Biochemistry and Behavior, 1978, 9, 597-602.

Gilden, L., \& Kozakiewicz, R. The effect of morphine on the EEG of the hypothalamus in the rat. Physiology \& Behavior, 1976, 16, 169-176.

KNIGGE, K. M. Adrenocortical response to immobilization in rats with lesion in hippocampus and amygdala, Federation Proceedings, 1961, 20, 185.

TENKE, C. E. Pontile synchronization of the hippocampal and lateral hypothalamic EEG: Spectral correlates of an evoked state. Unpublished doctoral dissertation, CityUniversity of New York, 1983.

(Manuscript accepted for publication October 26, 1983.) 